\title{
Shea Butter as a Viable Couplant for Ultrasound Imaging
}

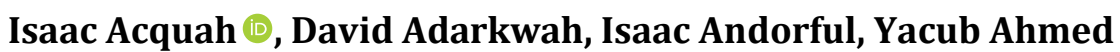

Biomedical Engineering Program, Kwame Nkrumah University of Science and Technology, Kumasi, Ghana

Correspondence to: Isaac Acquah, iacquah.soe@knust.edu.gh

Keywords: Medical Imaging, Ultrasound, Coupling Medium, Shea Butter

Received: December 5, 2018 Accepted: January 22, 2019 Published: January 25, 2019

Copyright $\odot 2019$ by authors and Scientific Research Publishing Inc.

This work is licensed under the Creative Commons Attribution International License (CC BY 4.0).

http://creativecommons.org/licenses/by/4.0/

\section{(c) (i) Open Access}

\section{ABSTRACT}

Ultrasound imaging remains one of the widespread diagnostic imaging tools. The commercial coupling gel for ultrasound imaging is quite expensive and not readily available in developing countries. However, shea butter, which is abundant locally and affordable, has suitable acoustic properties that makes it worthwhile for consideration as a coupling agent. Thus, shea butter was investigated alternate coupling medium for ultrasound imaging. In the study, ultrasound images were acquired using commercially available gel and shea butter. The image quality assessment of the images obtained using the gel (reference sample) and shea butter (reference sample) were performed using mean pixel intensity, mean square error (MSE), peak signal to noise ratio (PSNR) and structural similarity index matrix (SSIM) as image quality metrics. The resulting computed values of the MSE, PSNR and SSIM were respectively $40.1283 \pm 20.4941,32.7450 \pm 2.5722$ and $0.5934 \pm 0.1035$ (mean \pm standard deviation). The ranges of these metrics indicate that the images captured using gel and shea butter as couplants were comparable and supported the hypothesis that shea butter could be used as an ultrasound couplant. Using the Mann Whitney $U$ test as a test of significance with $\alpha$-value set at 0.05 , there was no significant difference in the mean pixel intensities between the two set of images. This inference further buttressed the proposition that shea butter could be used as an alternate coupling medium for ultrasound imaging.

\section{INTRODUCTION}

Medical ultrasound imaging remains invariably one of the commonest diagnostic imaging technique in hospitals and clinics [1]. The ubiquitous nature of ultrasound imaging has been attributed to its safety, good visualization and flexibility. It has been applied in obstetrics, breasts, genitourinary, musculoskeletal, pelvic and abdominal examinations $[2,3]$. This imaging modality uses high-frequency sound waves and the acoustic properties of body tissues to visualize internal structures of the human body [4]. Due to the fact that ultrasound images can be captured in real-time, the versatility of the imaging method is extended 
to capture both stationary and dynamic images of body organs [5].

During ultrasound imaging, a pulse of ultrasound is transmitted from an ultrasound transducer transverses body tissues of different acoustic properties, with a fraction of the ultrasound reflected as an echo. Ultrasound images are then generated from the acquisition and subsequent manipulation of the echoes over time. Image quality is dependent on the acoustic properties of tissues and intensity of ultrasound energy transmitted through the tissues. As ultrasound moves from one medium to another, there is maximum transmission of ultrasound energy if the media have approximately equal acoustic impedances [6]. On the contrary, a greater disparity in the impedance at the boundary between two media causes a tremendous reflection of ultrasound and consequently reducing the intensity of the ultrasound transmitted. The existence of an air layer between an ultrasound probe and skin creates an impedance mismatch at the skin-probe interface [7]. This mismatch can result in more than $99 \%$ reflection of ultrasound by air [8]. The fraction of transmitted ultrasound energy therefore is incapable of generating images of targeted organs. To minimize the mismatch and consequently enhance the transmission of ultrasound, a suitable coupling medium such as gel is applied on the skin prior to imaging to eliminate the air layer and improve impedance synergy. The exclusion of air by the coupling medium ensures maximum transmission of ultrasonic energy from transducers to test specimens and subsequently improve the quality of ultrasound images. A suitable coupling medium must be moderately viscous stay in place between the skin and probe. Additionally, the acoustic impedance of the coupling agent must match that of the interface tissue to permit maximum transmission of ultrasound energy, with negligible attenuation [7].

Commercially available ultrasound gel is the most commonly used coupling medium [9]. However, in Ghana and other parts of Africa, this gel is imported. Thus, the cost and availability of ultrasound gel in rural and deprived communities imposes significant constraints on the number and quality of scans available to clinicians even in settings with reliable working ultrasound scanners. More so, most medical scans and examinations are delayed due to the unavailability of ultrasound gel as a result of constant importation of the gel in most developing countries. The cost of Aquasonic commercial gel is about $\$ 50$ per bottle of 5 litres (Bio-Medical Instruments Inc, USA). The gel is even sold at higher prices locally due to the inclusion of import duties and profits of local vendors. Though this amount can be considered low in the developed countries, it is relatively expensive in many developing countries, where many live below the poverty line. This cost is invariably transferred to patients patronizing health facilities. Furthermore, some medical establishments tend to manage their scarcely available coupling media by utilizing them in inadequately small amounts and thus compromising quality scans for gel conservation. This development is alarming due to the high morbidity and mortality in obstetric and trauma cases reported in deprived communities, where ultrasound imaging has been extremely useful in-patient treatment and management $[10,11]$.

Some researchers have explored the possibility of making coupling agents from local materials or assessed different local materials for their suitability as coupling media $[10,12]$. Honey and shea butter were studied to evaluate their appropriateness for therapeutic ultrasound [13]. The transmissivity data for shea butter was promising.

Shea butter is derived from the processing nuts of shea trees (Vitellaria paradoxa) and has a myriad of applications including food, medicine and cosmetics [14, 15]. It has been used as edible oil and as a substitute of cocoa butter in chocolate products [16]. Further, shea butter, which has both anti-tumor and anti-inflammatory effects [17], presents itself as a potent moisturizer for patients suffering from atopic dermatitis [18].

Shea butter, which is abundant in West Africa [19], is biocompatible, viscous at normal body temperature and has acoustic impedance closer to that of skin $[14,15,20]$. These attributes, among others, make shea butter a viable candidate as a couplant for ultrasound imaging. The acoustic impedance of shea butter, $Z_{\text {shea }}$ calculated by the product of the density of shea butter $\left(=910 \mathrm{~kg} / \mathrm{m}^{3}\right)$ and speed of sound in shea butter $(=1450 \mathrm{~m} / \mathrm{s})$ [21] which gives $1.32 \times 10^{6}$ rayl. Given the acoustic impedance of the skin, $Z_{\text {skin }}$, is $1.99 \times 10^{6}$ rayl, the reflection coefficient, $R$, can be derived from Equation (1) [2]. 


$$
R=\left(\frac{Z_{\text {skin }}-Z_{\text {sheabutter }}}{Z_{\text {skin }}+Z_{\text {sheabutter }}}\right)^{2}
$$

Equation (1) yields a reflection coefficient of 0.04 . This implies that shea butter will allow up to $96 \%$ of ultrasonic energy to be transmitted into the body, which is sufficiently high to generate quality images for diagnosis.

In the attempt to find locally accessible alternative to the commercial gels, we investigated shea butter as an alternative coupling medium. We explored the properties of shea butter as a coupling medium, acoustic properties of shea butter and investigated its applicability as an alternate coupling agent based on image quality assessment indices. Images acquired using shea butter and commercial gel as couplants were compared using statistical measures.

\section{METHODOLOGY}

\subsection{Procedure}

Nine male students volunteered to participate in the study as subjects. Their ages were in the range 21 25 years (mean of 23). The purpose of the study and all research-related information were duly explained to the participants and their signed informed consent were obtained. The study was also given approval by the Ethics Review Committee of the Kwame Nkrumah University of Science and Technology, Ghana. With the help of qualified sonographers at the University Hospital, Kwame Nkrumah University of Science and Technology (Kumasi, Ghana) ultrasound images of the spleen, kidney, thyroid and gall bladder of participants were captured using hypoallergenic ultrasound gel (Microtech Medical, UK) and shea butter (from the local market, Kumasi, Ghana) as coupling media under the same room conditions. Only one couplant was used at a time. The sonographers were careful to ensure that the same organ was imaged twice with ultrasound gel and shea butter, respectively, while the subject laid in supine position on an ultrasound examination table. The ultrasound probe and area of contact of the couplant with the skin were thoroughly cleaned after each scan. The ultrasound machine, Philips ClearVue 350, was employed in the acquisition of the images for the study. For each participant, four (4) or five (5) pairs of images were captured. In all, a total of forty pairs of images were acquired for further analysis.

\subsection{Treatment of Data}

Soft copies of the ultrasound images acquired using commercial gel and shea butter by the ultrasound machine were retrieved and processed in MATLAB (MathWorks Inc., United States) to compute mean pixel intensity, mean square error (MSE), peak signal to noise ratio (PSNR) and structural similarity index matrix (SSIM). These metrics served as the indices for the comparison of the two types of images and were calculated according to Equations (2) to (5) [22, 23].

$$
\begin{gathered}
\text { Mean pixel intensity }=\frac{1}{M N}=\sum_{i=0}^{M} \sum_{j=0}^{N}|X(i, j)| \\
\qquad \text { MSE }=\frac{1}{M N}=\sum_{i=0}^{M} \sum_{j=0}^{N}(x(i, j)-y(i, j)) \\
\text { PSNR }=10 \log _{10} \frac{\operatorname{MaxI}^{2}}{M S E} \\
\operatorname{SSIM} \frac{2\left(\mu_{x}+\mu_{y}\right)\left(2 \sigma_{x y}\right)}{2\left(\mu_{x}+\mu_{y}\right)\left(\sigma_{x}^{2}+\sigma_{y}^{2}\right)}
\end{gathered}
$$

where, 


$$
\begin{gathered}
\mu_{x}=\frac{1}{N} \sum_{i=1}^{N} x_{i}, \mu_{y}=\frac{1}{N} \sum_{i=1}^{N} y_{i} \\
\sigma_{x}=\sqrt{\frac{1}{N-1} \sum_{i=1}^{N}\left(x_{i}-\mu_{x}\right)^{2}}, \sigma_{y}=\sqrt{\frac{1}{N-1} \sum_{i=1}^{N}\left(y_{i}-\mu_{y}\right)^{2}} \\
\sigma_{x y}=\frac{1}{N-1} \sum_{i=1}^{N}\left(x_{i}-\mu_{x}\right)\left(y_{i}-\mu_{y}\right)
\end{gathered}
$$

$M=$ number of pixels in the horizontal direction

$N=$ number of pixels in the vertical direction

$x(i, j)=$ reference image

$y(i, j)=$ test image

MaxI $^{2}=$ maximum pixel value

The calculated measures served as indices for an objective assessment of image quality acquired using the different couplants. Other statistical information and graphs were derived using Excel (Microsoft) data analysis toolbox and Origin 8 (OriginLab Corporation, United States). A Mann-Whitney $U$ (calculated using Equation (6)) test was also used to determine whether the mean image pixel intensities vary significantly between the sample pairs, with alpha $(\alpha)$ set at 0.05 .

$$
U=\min \left(U_{1}, U_{2}\right)
$$

where

$$
\begin{gathered}
U_{1}=n_{1} n_{2}+\frac{n_{1}\left(n_{1}+1\right)}{2}-R_{1} \\
U_{2}=n_{1} n_{2}+\frac{n_{2}\left(n_{1}+1\right)}{2}-R_{2}
\end{gathered}
$$

$R_{1}=$ sum of rank for gel

$R_{2}=$ sum of rank for shea butter

$n_{1}=n_{2}=$ number of samples $=40$

\section{RESULTS AND DISCUSSION}

Quality medical images are important for diagnosis, treatment, management and monitoring of diseases and trauma. However, images acquired through medical imaging techniques prone to distortion from artifacts that can mask significant features in images. Artifacts may arise from the real source and processing steps [24]. There exist numerous algorithms to remove or minimize artifacts in the processing stages [25-27]. Therefore, in order to capture quality images for medical analysis, the real source must be thoroughly prepared prior to image acquisition. Application of a couplant preceding ultrasound imaging is thus an important stage.

In this study, images acquired were evaluated by objective assessment of image quality. We analysed the mathematical deviations between the referenced images (with ultrasound gel) and the test images (with shea butter) by calculating the mean pixel intensities, MSE, PSNR and SSIM between images using gel and shea butter as couplants respectively. MSE, PSNR and SSIM are widely used statistical measures in image quality evaluation describe the level of similarity between images [22, 23, 28].

The ultrasound images of various organs (gall bladder, kidney, thyroid, spleen and liver) taken using gel and shea butter are displayed in Figure 1.

The mean pixel intensity represents the contribution of all the pixels in an image. A higher value indicates a better image contrast [22]. As evident from Figure 2, there exists some level of variation in the mean pixel intensities between the images captured using gel and shea butter as computed and obtained 
from MATLAB. Clearly, the images acquired shea butter had greater mean pixel intensities compared to that of obtained with ultrasound gel. Whether these differences are significant or not, requires a test of significance to determine.

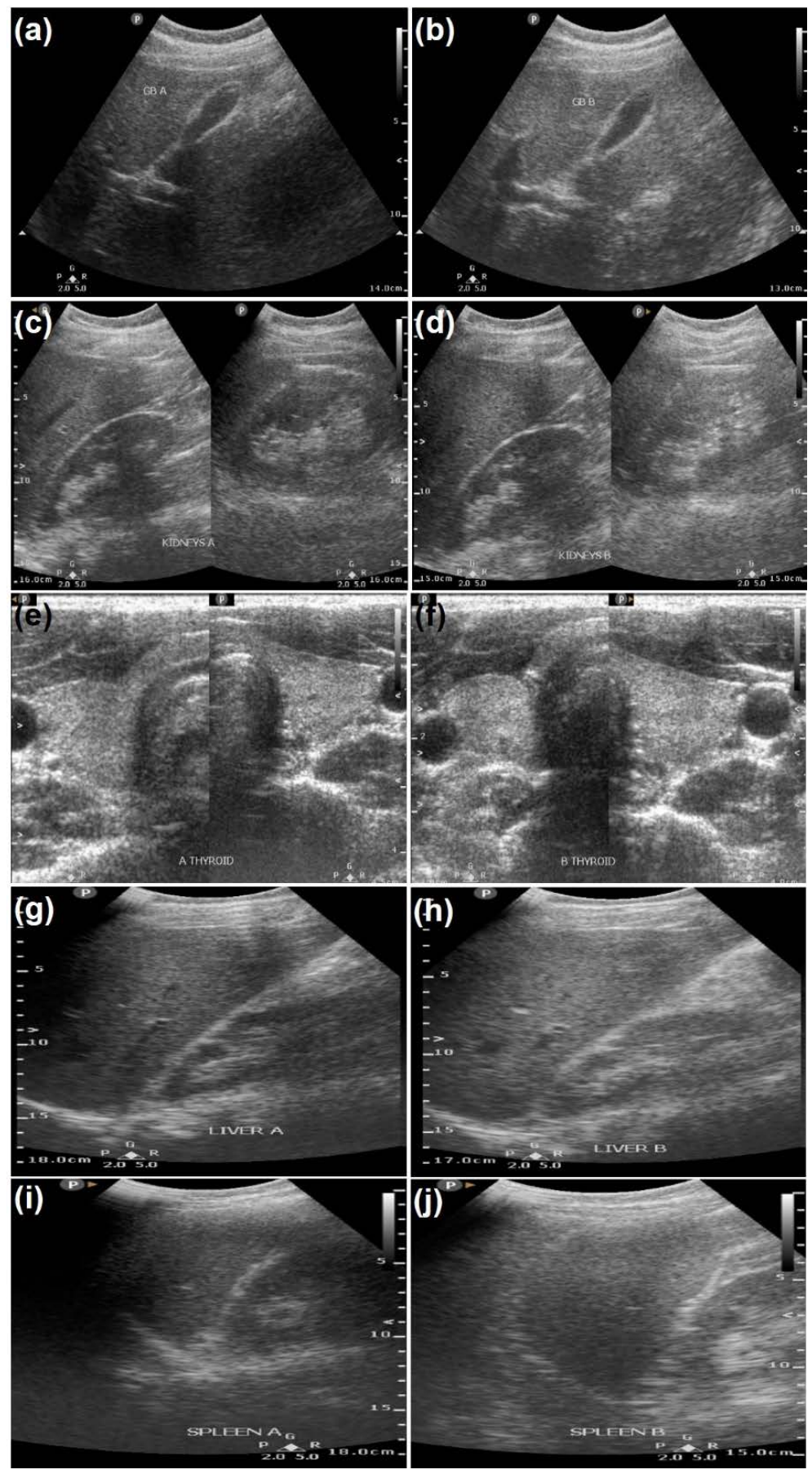

Figure 1. Images taken using gel (left) and shea butter (right): (a) gall bladder taken with gel, (b) gall bladder taken with shea butter, (c) kidneys (left and right sides) taken with gel, (d) kidneys (left and right sides) taken with shea butter (e) thyroid taken with gel, (f) thyroid taken with shea butter, (g) liver taken with gel, (h) liver taken with shea butter, $i)$ spleen taken with gel, $(j)$ spleen taken with shea butter. 


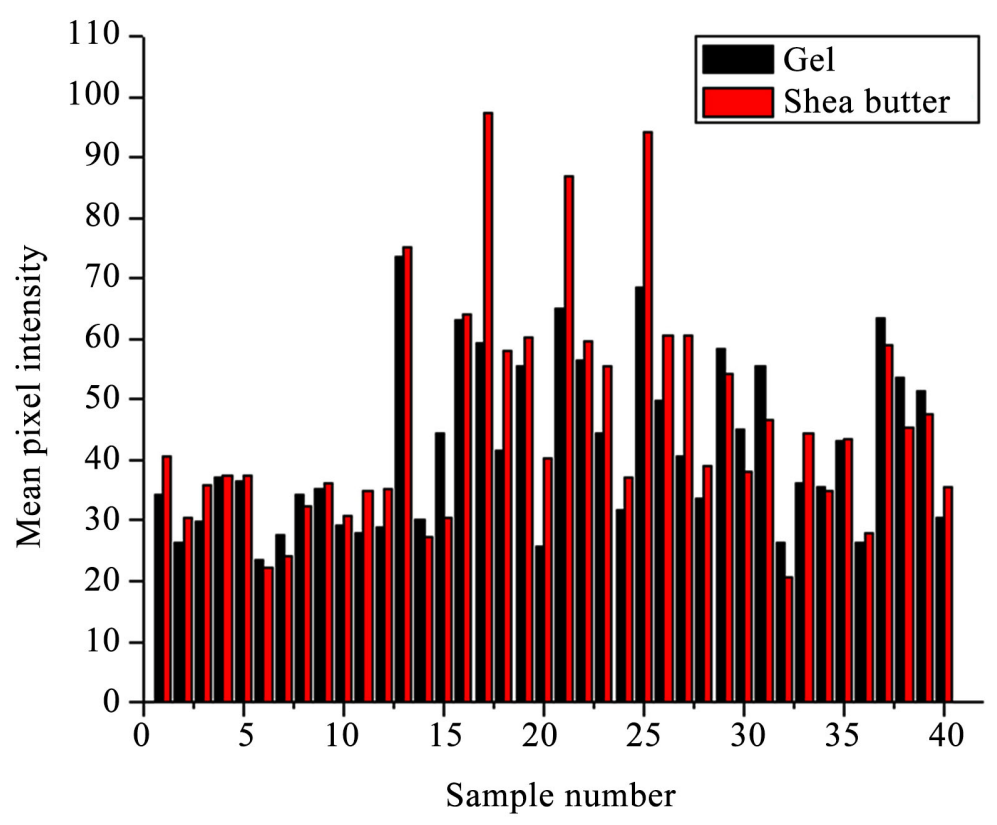

Figure 2. Mean pixel intensities of images.

Mean square error (MSE) and peak signal to noise ratio (PSNR) are considered excellent measures in optimization applications in image quality evaluation when comparing images $[23,29]$. MSE is a dispersion metric that measures the difference between a reference image (with gel) and a test image (with shea butter). However, these metrics do not sufficiently predict how well the distortion between the two images can be seen especially images with large luminance variations [30]. This limitation was catered for by the introduction of structural similarity index metric (SSIM). SSIM provides a better approximation of perceived image distortion and can be applied to assess the quality of any image [22].

The statistics obtained from the mean pixel intensities (PI), MSE, PSNR and SSIM values from the ultrasound images have been tabulated in Table 1. An MSE score lies in the interval of 0 to $\infty$. If the calculated MSE index approaches $\infty$, the difference between the images is considered huge. When the MSE score is low, it depicts a small difference between the images compared and a high visual quality [31]. The mean MSE was found to be 40.1283 and was subsequently considered low enough to represent a high level of similarity between the images acquired using the commercial gel and shea butter respectively. A PSNR value, which reveals the distortion present between the sample images, derives from the MSE (Equation (4)). The PSNR values computed were in the standard range of $30-40 \mathrm{~dB}$ [22]. The PSNR value of 32.7450 \pm 2.5722 , lies in the standard PSNR range and signifies less distortion between image samples. However, the greater the value of PSNR in the standard range $(30-40 \mathrm{~dB})$, the better the image quality [22]. For better clinical diagnosis, PSNR index of $35 \mathrm{~dB}$ is recommended [32].

SSIM sheds lights on how well the perceived structural information can be obtained from an image. The SSIM values lies in the interval of -1 to 1 , with the best value being 1 [33]. With a mean SSIM value of 0.59305 , higher than the average, corroborates that the perceived structural information can be obtained from the image samples.

The difference between averaged mean pixel intensities for ultrasound gel and shea butter was 4.0557 (Table 1). However, the absolute value of this difference is not enough to sufficiently conclude whether the two sets of images differ significantly. Moreover, the differences may be due to factors other than the couplants, such as bowel movements and cleaning of probe. Therefore, we employed the Mann-Whitney $U$ test as a test of significance to verify if the difference deduced was significant or not. The Mann-Whitney $U$ test, a nonparametric equivalent of student's t-test, allows two populations to be compared without making the assumption that the values obtained are normally distributed [34]. The mean pixel intensities obtained for the images using ultrasound gel and shea butter represent the Utest datafor comparison. 
Table 1. Statistical measures.

\begin{tabular}{cccccc}
\hline Statistic & Mean PI (Gel) & Mean PI (Shea butter) & MSE & PSNR (dB) & SSIM \\
\hline Mean & 41.9783 & 46.0340 & 40.1283 & 32.7450 & 0.5934 \\
Standard Error & 2.2023 & 2.9341 & 3.2404 & 0.4067 & 0.0164 \\
Standard Deviation & 13.9285 & 18.5560 & 20.4941 & 2.5722 & 0.1035 \\
Sample Variance & 194.0043 & 344.3234 & 420.0082 & 6.6162 & 0.0107 \\
\hline
\end{tabular}

The null hypothesis is that there is no significant difference between the mean pixel intensities of the images acquired using gel and shea butter as coupling agents. The alternative hypothesis stipulates that mean pixel intensities for the images obtained using gel differs significantly from the mean pixel intensity distribution for images acquired using shea butter as couplant. For the computed Mann-Whitney $U$ of 696 , a z-score of -0.99593 corresponding to a p-value of 0.31732 was obtained. Since, the p-value was greater than the set $\alpha$-value of 0.05 , the null hypothesis cannot be rejected with a significance level of 0.95 . This clearly indicates that there is no significant difference between the two mean pixel intensities with a $95 \%$ confidence level. Thus, shea butter, which is readily available and cost effective could be used as an alternate coupling medium for ultrasound imaging.

It is worth noting that none of the subjects complained of any allergic reaction to any other couplants during the study.

\section{CONCLUSION}

In this study, we set out to investigate the suitability of shea butter as a coupling medium for ultrasound imaging due to the fact that conventional gel is quite expensive and not readily available locally. The low cost, abundance and physico-chemical properties of shea butter make it adaptable for high transitivity of ultrasound emission to internal organs to be imaged. Subsequently, ultrasound images of internal organs were captured using shea butter as an alternative to the commercial gel. Comparing the quality of the set of ultrasound images obtained using gel (reference) and shea butter as coupling agents, the MSE, PSNR and SSIM scores proved that the quality of shea butter images was of acceptable quality. More so, the Mann Whitney $U$ test proved that there is no significant difference between the mean pixel intensities of the set of images acquired using gel and shea butter. Thus, shea butter could be employed as a substitute to commercial gel for ultrasound imaging though further research is required to assess the optimal conditions for the application of shea butter as a couplant.

\section{CONFLICTS OF INTEREST}

The authors declare no conflicts of interest regarding the publication of this paper.

\section{REFERENCES}

1. Steinmetz, J. and Berger, J. (1999) Ultrasonography as an Aid to Diagnosis and Treatment in a Rural African Hospital: A Prospective Study of 1119 Cases. The American Journal of Tropical Medicine and Hygiene, 60, 119-123. https://doi.org/10.4269/ajtmh.1999.60.119

2. Wilhjelm, J.E., AndreasIllum, M.K. and Trier, O. (2013) Medical Diagnostic Ultrasound Physical Principles and Imaging. Biomedical Engineering, DTU Elektro Technical University of Denmark.

3. Spencer, J.K. and Adler, R.S. (2008) Utility of Portable Ultrasound in a Community in Ghana. Journal of Ultrasound in Medicine, 27, 1735-1743. https://doi.org/10.7863/jum.2008.27.12.1735

4. Monchalin, J.-P. (1991) Optical Generation and Detection of Ultrasound. In: Leroy, O. and Breazeale, M.A., Eds., Physical Acoustics, Springer, Boston, MA, 65-76. https://doi.org/10.1007/978-1-4615-9573-1_7 
5. Hodges, P., Pengel, L., Herbert, R. and Gandevia, S. (2003) Measurement of Muscle Contraction with Ultrasound Imaging. Muscle and Nerve, 27, 682-692. https://doi.org/10.1002/mus.10375

6. Theobald, P., Zeqiri, B. and Avison, J. (2008) Couplants and Their Influence on AE Sensor Sensitivity. Journal of Acoustic Emission, 26, 91-97.

7. Poltawski, L. and Watson, T. (2007) Relative Transmissivity of Ultrasound Coupling Agents Commonly Used by Therapists in the UK. Ultrasound in Medicine and Biology, 33, 120-128. https://doi.org/10.1016/j.ultrasmedbio.2006.07.026

8. Hasan, A.A. and Makhlouf, H.A. (2014) B-lines: Transthoracic Chest Ultrasound Signs Useful in Assessment of Interstitial Lung Diseases. Annals of Thoracic Medicine, 9, 99. https://doi.org/10.4103/1817-1737.128856

9. Draper, D.O., Rigby, J. and Wells, A. (2014) Comparison of the Gel-SHOT versus Traditional Ultrasound Gel to Raise Tissue Temperature. Athletic Training and Sports Health Care, 6, 273-279. https://doi.org/10.3928/19425864-20141128-02

10. Salmon, M., Salmon, C., Bissinger, A., Muller, M.M., Gebreyesus, A., Geremew, H., Wendell, S., Azaza, A., Salumu, M. and Benfield, N. (2015) Alternative Ultrasound Gel for a Sustainable Ultrasound Program: Application of Human Centered Design. PloS One, 10, e0134332. https://doi.org/10.1371/journal.pone.0134332

11. Groen, R.S., Leow, J.J., Sadasivam, V. and Kushner, A.L. (2011) Indications for Ultrasound Use in Low- and Middle-Income Countries. Tropical Medicine and International Health, 16, 1525-1535. https://doi.org/10.1111/j.1365-3156.2011.02868.x

12. Casarotto, R.A., Adamowski, J.C., Fallopa, F. and Bacanelli, F. (2004) Coupling Agents in Therapeutic Ultrasound: Acoustic and Thermal Behavior. Archives of Physical Medicine and Rehabilitation, 85, 162-165. https://doi.org/10.1016/S0003-9993(03)00293-4

13. Adegoke, B.O. and Edeni, K. (2009) Transmissivity of Some Local Couplants and Phonophoretic Drugs. Journal of Nigeria Society of Physiotherapy, 14, 18-23.

14. Chalfin, B. (2004) Shea Butter Republic: State Power, Global Markets, and the Making of an Indigenous Commodity. Routledge, New York. https://doi.org/10.4324/9780203496534

15. Honfo, F.G., Akissoe, N., Linnemann, A.R., Soumanou, M. and Van Boekel, M.A. (2014) Nutritional Composition of Shea Products and Chemical Properties of Shea Butter: A Review. Critical Reviews in Food Science and Nutrition, 54, 673-686. https://doi.org/10.1080/10408398.2011.604142

16. Nahm, H.S., Juliani, H.R. and Simon, J.E. (2012) Effects of Selected Synthetic and Natural Antioxidants on the Oxidative Stability of Shea Butter (Vitellaria paradoxa subsp. paradoxa). Journal of Medicinally Active Plants, 1, 69-75.

17. Akihisa, T., Kojima, N., Kikuchi, T., Yasukawa, K., Tokuda, H., Masters, E.T., Manosroi, A. and Manosroi, J. (2010) Anti-Inflammatory and Chemopreventive Effects of Triterpene Cinnamates and Acetates from Shea Fat. Journal of Oleo Science, 59, 273-280. https://doi.org/10.5650/jos.59.273

18. Sirikudta, W., Kulthanan, K., Varothai, S. and Nuchkull, P. (2013) Moisturizers for Patients with Atopic Dermatitis: An Overview. Journal of Allergy and Therapy, 4, 1-6. https://doi.org/10.4172/2155-6121.1000143

19. Goreja, W. (2004) Shea Butter: The Nourishing Properties of Africa's Best-Kept Natural Beauty Secret. TNC International Inc.

20. Okullo, J.B.L., Omujal, F., Agea, J., Vuzi, P., Namutebi, A., Okello, J. and Nyanzi, S. (2010) Physico-Chemical Characteristics of Shea Butter (Vitellaria Paradoxa CF Gaertn.) Oil from the Shea District of Uganda. African Journal of Food, Agriculture, Nutrition and Development, 10, 2070-2084.

https://doi.org/10.4314/ajfand.v10i1.51484

21. Azhari, H. (2010) Basics of Biomedical Ultrasound for Engineers. John Wiley \& Sons, Hoboken, 313-314. 
https://doi.org/10.1002/9780470561478

22. Rajkumar, S. and Malathi, G. (2016) A Comparative Analysis on Image Quality Assessment for Real Time Satellite Images. Indian Journal of Science and Technology, 9, 1-11. https://doi.org/10.17485/ijst/2016/v9i34/96766

23. Wang, Z. and Bovik, A.C. (2009) Mean Squared Error: Love It or Leave It? A New Look at Signal Fidelity Measures. IEEE Signal Processing Magazine, 26, 98-117. https://doi.org/10.1109/MSP.2008.930649

24. Mredhula, L. and Dorairangasamy, M. (2013) An Extensive Review of Significant Researches on Medical Image Denoising Techniques. International Journal of Computer Applications, 64, 1-12.

25. Wang, W., Zhu, L., Qin, J., Chui, Y.-P., Li, B.N. and Heng, P.-A. (2014) Multiscale Geodesic Active Contours for Ultrasound Image Segmentation Using Speckle Reducing Anisotropic Diffusion. Optics and Lasers in Engineering, 54, 105-116. https://doi.org/10.1016/j.optlaseng.2013.10.003

26. Bhateja, V., Srivastava, A., Singh, G. and Singh, J. (2014) A Modified Speckle Suppression Algorithm for Breast Ultrasound Images Using Directional Filters. Proceedings of the 48 th Annual Convention of Computer Society of India, Vol. 2, Visakhapatnam, 13-15 December 2013, 219-226. https://doi.org/10.1007/978-3-319-03095-1_24

27. Kachouie, N.N. (2008) Anisotropic Diffusion for Medical Image Enhancement. International Journal of Image Processing, 4, 436-443.

28. Wang, Z., Bovik, A.C., Sheikh, H.R. and Simoncelli, E.P. (2004) Image Quality Assessment: From Error Visibility to Structural Similarity. IEEE Transactions on Image Processing, 13, 600-612.

https://doi.org/10.1109/TIP.2003.819861

29. Mohammadi, P., Ebrahimi-Moghadam, A. and Shirani, S. (2014) Subjective and Objective Quality Assessment of Image: A Survey. arXiv:1406.7799

30. Gadkari, D. (2004) Image Quality Analysis Using GLCM. MSc Thesis, University of Central Florida, Orlando.

31. Dosselmann, R. and Yang, X.D. (2011) A Comprehensive Assessment of the Structural Similarity Index. Signal, Image and Video Processing, 5, 81-91. https://doi.org/10.1007/s11760-009-0144-1

32. Garawi, S., Istepanian, R.S. and Abu-Rgheff, M.A. (2006) 3G Wireless Communications for Mobile Robotic Tele-Ultrasonography Systems. IEEE Communications Magazine, 44, 91-96.

https://doi.org/10.1109/MCOM.2006.1632654

33. Istepanian, R., Philip, N., Martini, M., Amso, N. and Shorvon, P. (2008) Subjective and Objective Quality Assessment in Wireless Teleultrasonography Imaging. Proceeding of the 30 th Annual International Conference of the IEEE EMBS, Vancouver, 20-25 August 2008, 5346-5349.

34. Rosner, B. and Grove, D. (1999) Use of the Mann-Whitney U-Test for Clustered Data. Statistics in Medicine, 18, 1387-1400. https://doi.org/10.1002/(SICI)1097-0258(19990615)18:11<1387::AID-SIM126>3.0.CO;2-V 